\title{
About the kinetic energy of mutual motion of synchronous machine rotors under disturbance in a large power system
}

\author{
Nikolai I. Voropai ${ }^{1,}{ }^{*}$, Sergei V. Stashkevich ${ }^{1}$ \\ ${ }^{1}$ Melentiev Energy Systems Institute, 130 Lermontov str., Irkutsk, Russia
}

\begin{abstract}
The article shows the analysis of the components of the mutual motion of the synchronous machines rotors in the transient process under disturbance conditions. It is shown that the representation of the original EPS can be represented by a two-machine equivalent, relative to the section in which stability is violated.
\end{abstract}

\section{Introduction}

In large electric power systems (EPS) under disturbances electromechanical transient processes occur, whose unfavorable course creates stability problems in EPS $[1,2]$. Depending on the structure and properties of EPSs, several types of their stability are distinguished, one of which is "rotor angle stability", which is typical for large EPSs with long transmission lines. This type of stability is determined by the nature of the rotors mutual motion in synchronous machines during the electromechanical transition after the disturbance.If the stability of the EPS is not disturbed, then synchronous mutual oscillations of the rotors of synchronous machines occur in a limited range of changes in the angles of the rotors. In case of the stability violation in a power system along some section of the electrical network, two groups of synchronous machines are initially formed, within each of which the synchronous motion of their rotors is maintained, and between the groups there exists an unlimited increase in the mutual angles of the rotors of the synchronous machines located in different groups, i.e. asynchronous mode that is interrupted by the automatic elimination of the asynchronous mode (AEAM). The word 'initial' here means that after the first noted violation of the EPS stability, one or more violations of stability along other sections can subsequently occur, and as a result of it a multifrequency asynchronous mode will arise.

At the same time, it is important to emphasize that this first violation of EPS stability occurs in the weakest section of the electrical network during the transient process moment. The next violation of the power system stability will also occur in the weakest section, which is characteristic for changed circuit of the electrical network after the elimination of the first asynchronous mode by the action of AEAM. The well-known features of electromechanical transient processes in EPS in cases of "rotor angle stability" problems are considered here to show that in such situations the mechanical component plays the defining role in the general composition of electromechanical properties of EPS, it is characterized by the inertia of the rotating masses of the synchronous machines rotors. Consequently, it is logical to use the basic provisions of mechanics for the analysis of electromechanical transient processes and "rotor angle stability" of EPS in this case, [3], especially since there is a successful positive experience in this regard [4].

From the point of view of the basic provisions of mechanics, the rotors of synchronous machines receive kinetic energy as a result of the disturbance, which is realized through the oscillations of their mutual angles.

The kinetic energy is determined by the Lagrange function and in the case under consideration has the form [4]:

$$
K=\sum_{i=}^{n} \frac{T_{J i} s_{i}^{2}}{2}
$$

where $T_{J i}$ is the constant of inertia of the synchronous machine rotor $i ; \mathrm{s}_{i}$ is the slip of the rotor of the synchronous machine $i$ relative to the synchronous axis; $n$ is the number of synchronous machines in the EPS.

\section{Proposed test circuit}

It is of interest to analyze the features of the structure of the kinetic energy of the synchronous machines mutual motion of rotors as a result of disturbance for two typical cases: maintaining and violation of the EPS stability. To do this, we will carry out necessary calculations in relation to the four-machine test circuit of the EPS, given in [2]. The test circuit is shown in Fig. 1, its parameters are in Tables 1-3. 


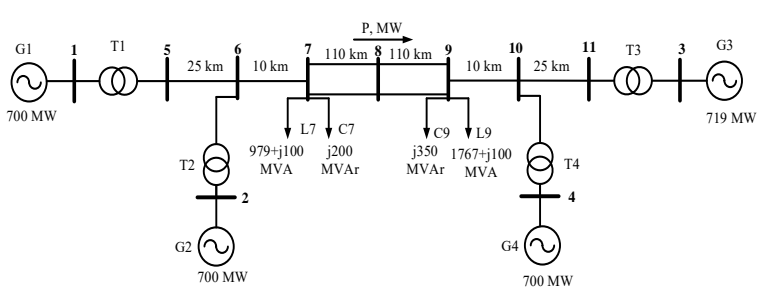

Fig. 1. EPS test circuit

Table 1. Generator parameters

\begin{tabular}{|c|c|c|c|c|}
\hline $\begin{array}{c}\text { Equipment/ } \\
\text { parameters }\end{array}$ & $\mathbf{G 1}$ & $\mathbf{G 2}$ & $\mathbf{G 3}$ & $\mathbf{G 4}$ \\
\hline$\dot{X}^{\prime}{ }_{d}, \Omega$ & $j 17,63$ & $j 17,63$ & $j 17,63$ & $j 17,63$ \\
\hline$\dot{Y}^{\prime}{ }_{d}, S$ & $-j 0,057$ & $-j 0,057$ & $-j 0,057$ & $-j 0,057$ \\
\hline$\dot{E}^{\prime}{ }_{d}, k V$ & $236,9 e^{j 20,2}$ & $236,9 e^{j 10,5}$ & $236,9 e^{-j 6,8}$ & $236,9 e^{-j 17}$ \\
\hline
\end{tabular}

Table 2. Parameters of transformers, loads

\begin{tabular}{|c|c|c|}
\hline $\begin{array}{c}\text { Equipment/ } \\
\text { parameters }\end{array}$ & $\dot{X}_{T}, \Omega$ & $\dot{Y}_{T}, S$ \\
\hline $\mathbf{T}_{\mathbf{1 - 5}}$ & $j 8,82$ & $-j 0,11$ \\
\hline $\mathbf{T}_{\mathbf{2 - 6}}$ & $j 8,82$ & $-j 0,11$ \\
\hline $\mathbf{T}_{\mathbf{3 - 1 1}}$ & $j 8,82$ & $-j 0,11$ \\
\hline $\mathbf{T}_{\mathbf{4 - 1 0}}$ & $j 8,82$ & $-j 0,11$ \\
\hline $\mathbf{L 7}$ & 54,13 & $0,0183+$ \\
& $-j 5,59$ & $j 0,0019$ \\
\hline $\mathbf{L 9}$ & 29,84 & $0,033+$ \\
& $-j 1,69$ & $j 0,0019$ \\
\hline $\mathbf{C 7}$ & $j 264,5$ & $-j 0,0038$ \\
\hline $\mathbf{C 9}$ & $j 151,14$ & $-j 0,0066$ \\
\hline
\end{tabular}

Table 3. Power transmission line parameters

\begin{tabular}{|c|c|c|c|}
\hline $\begin{array}{c}\text { Equipment/ } \\
\text { parameters }\end{array}$ & $\dot{Z}_{i j}, \Omega$ & $\dot{Y}_{i j}, S$ & $\frac{\dot{Y}_{C i j}}{2}, 10^{-6} \cdot S$ \\
\hline $\mathbf{L 5 - 6}$ & $1,32+j 13,23$ & $0,0075-j 0,0748$ & $j 41,66$ \\
\hline $\mathbf{L}_{6-7}$ & $0,529+j 5,29$ & $0,0187-j 0,1872$ & $j 16,67$ \\
\hline $\mathbf{L}_{7-8}$ & $2,9+j 29,1$ & $0,0034-j 0,034$ & $j 363,6$ \\
\hline $\mathbf{L 8 - 9}$ & $2,9+j 29,1$ & $0,0034-j 0,034$ & $j 363,6$ \\
\hline $\mathbf{L 9 - 1 0}$ & $0,529+j 5,29$ & $0,0187-j 0,1872$ & $j 16,67$ \\
\hline $\mathbf{L}_{\mathbf{1 0 - 1 1}}$ & $1,32+j 13,23$ & $0,0075-j 0,0748$ & $j 41,66$ \\
\hline
\end{tabular}

We consider the problem of determining the limiting transient stability of the flow along section 8-9, measured from the side of node 9 , for a given disturbance on the tap at node 7 . To do this, a program for calculating the electromechanical transient processes in time, developed by Matlab, is used, in which the classical model of the EPS dynamics is implemented, taking into account the assumptions generally accepted for this model [1, 2]. Since, as noted above, in this study, the mechanical component plays the defining role in the composition of the electromechanical properties of the EPS, the electrical component of the system properties should be taken into account approximately, at the level presented in the classical model of the EPS dynamics.

\section{Case studies}

First of all, attention should be paid to the nature of mutual motion of the rotors angles in synchronous machines in the first two calculations, where Fig. 2 illustrates the nature of the transient process while maintaining the EPS stability, and Fig. 3 shows when the system stability is disturbed. At the same time, in both cases, the pre-emergency mode and, accordingly, the flow through section $8-9$, are very close, the disturbance is the same, and therefore, the value of the kinetic energy imparted by the disturbance is the same (up to the difference in pre-emergency modes).

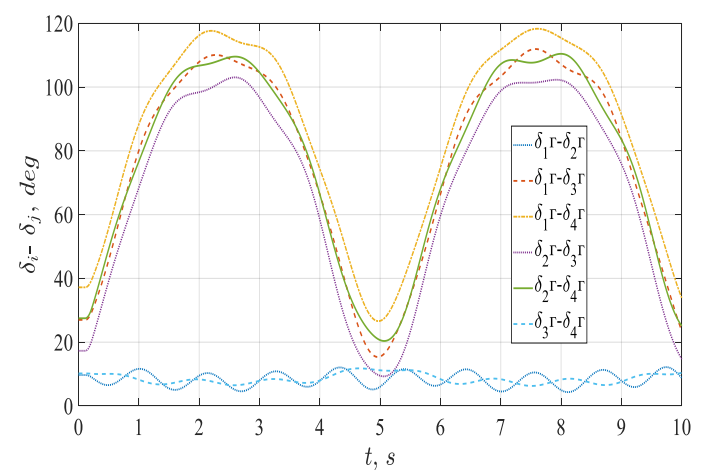

Fig. 2. Change of mutual angles of generators rotors with power flow in the section $8-9-299.8 \mathrm{MW}$

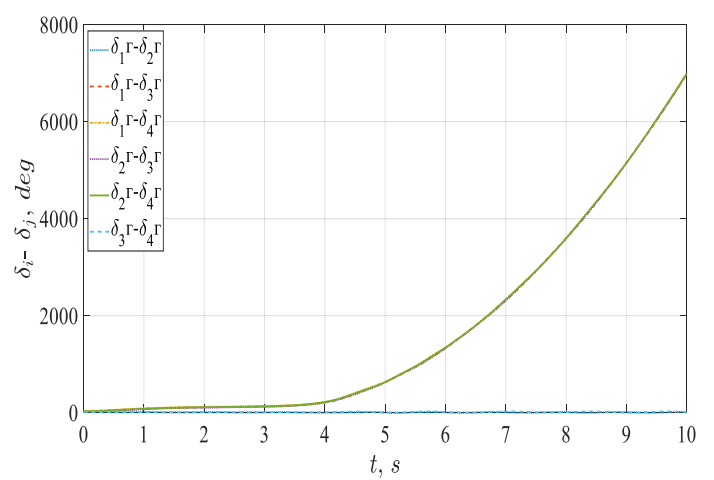

Fig. 3. Change of mutual angles of generators rotors with power flow in the section $8-9-304.4 \mathrm{MW}$

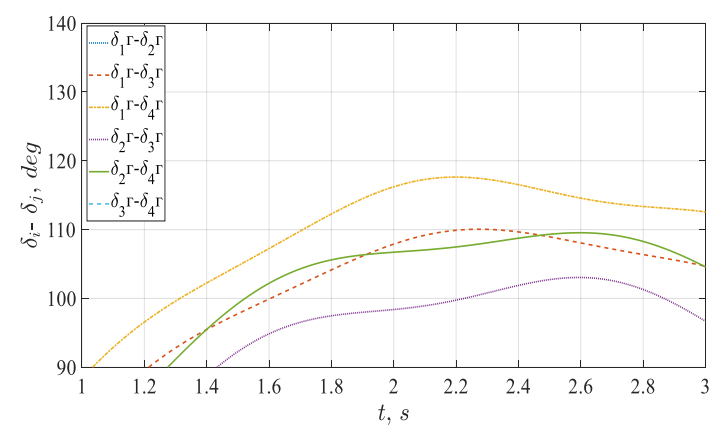

a) 


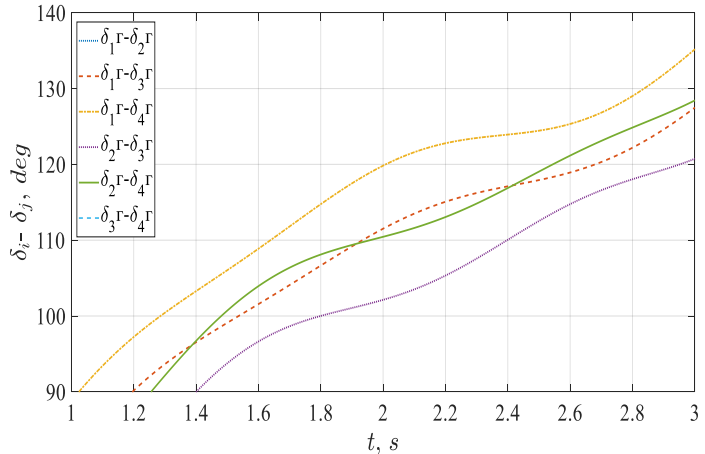

b)

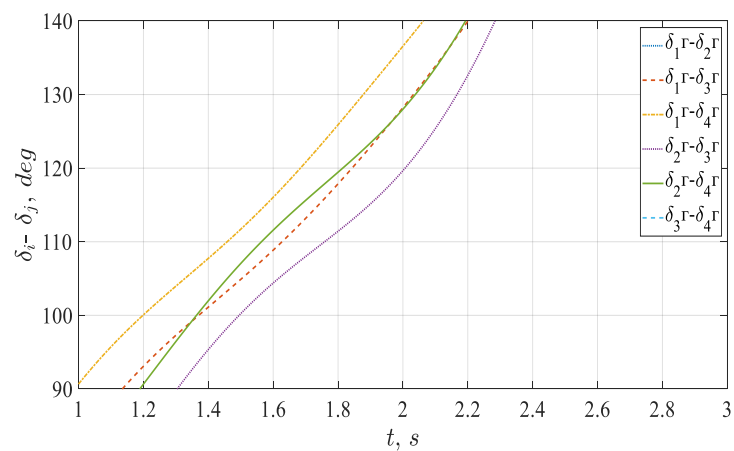

c)

Fig. 4. The nature of the mutual angles movement of the synchronous machines rotors while maintaining and breaking stability (cut from 1 to 3 seconds): a) crosssection of $8-9-299.8 \mathrm{MW}$; b) cross-section of $8-9-$ 304.4 MW; c) cross-section of 8-9-321 MW

In order to understand the nature of the motion synchronous machines rotors during disturbance more specifically and to make a confident comparison of various situations, Fig. 4 shows sections of the graphs for the mutual angles motion of synchronous machines rotors to consider both cases in the range from 1 to 3 seconds at the same scale (Fig. $4 \mathrm{a}$ and $4 \mathrm{~b}$ ). In the group of generators with large mutual oscillations (Figure 2), it can be seen that the amplitude of oscillations in case $b$ (violation of the stability of the EPS and the beginning of the asynchronous mode) is less than in case a (maintenance of the system stability). If we consider a more severe case of violation of the EPS stability with an increased flow of up to $321 \mathrm{MW}$ over the cross section 8-9, then we note that there are practically no mutual oscillations in the considered group of synchronous machines (Fig. 4c).

The nature of the processes under consideration allows us to draw the following conclusions:

1) In the case of maintaining the EPS stability, the kinetic energy determined by the disturbance is completely spent on the mutual oscillations of the angles of the synchronous machines rotors.

2) When the stability of the system is disturbed, the main part of the kinetic energy is spent on the arisen asynchronous mode, while the remaining parts of the kinetic energy in the asynchronously moving subsystems are much less than the total value of the kinetic energy, therefore, the mutual oscillations of the rotors of synchronous machines in the subsystems are much less than the mutual oscillations of the rotors of the same synchronous machines in the case of maintaining the EPS stability.

The formulated considerations correspond to the principle of the least action known in mechanics (Hamilton's principle) or the law of conservation of energy in a conservative system [4]. Taking into account the assumptions formulated above about the defining role of the mechanical properties of the system in the electromechanical transient processes of EPS, it seems possible to extend the law of conservation of energy to a non-conservative system, which is the classical model of EPS dynamics.

It is advisable to pay attention to the fact, given in clause 2, about a decrease in the intensity for mutual oscillations of the synchronous machines rotors in asynchronously diverging subsystems when the stability of the EPS is disturbed. In the limiting case, in the absence of such oscillations, one can speak about the motion coherence of the synchronous machines rotors (the equivalent term 'in-phase motion' was used in the 20th century in the Russian-language literature, [5]). The coherence of motion is the defining condition for the EPS equivalence (representation of a group of coherently moving synchronous machines by one equivalent synchronous machine) with a minimum error, within the limit of zero, at the ideal motion coherence, since the EPS dynamics model is nonlinear.

Thus, using the equivalence, for cases of violation of the EPS stability, an equivalent two-machine model of the dynamics of the system can be formed with acceptable accuracy; the classical method of areas is appropriately applied for the assessment of its stability [1, 2].

Considering the fact that the motion coherence of synchronous machines in subsystems is typical for cases of stability violation, an assessment of the stability of a complex EPS using a two-machine equivalent can be constructed from an obviously unstable initial state, which is characterized by loading a critical section of the electrical circuit in terms of stability, step by step reducing the load up to the limiting cross-sectional stability. Taking into account the simplicity of the area method, the time spent on determining the flow limit in terms of stability will be significantly less than it would be required when using the methods of numerical integration of the differential equations for dynamics complete model of the complex multi-machine EPS.

\section{Conclusion}

The analysis of the kinetic energy components of the mutual motion of the synchronous machines rotors in the transient process under disturbance conditions shows that when the stability of the EPS is disturbed, the kinetic energy of the mutual motion of the synchronous machines rotors is mainly spent on the asynchronous divergence of the synchronous machines rotors. At the same time, mutual oscillations of rotors inside asynchronously diverging groups of synchronous machines practically do not occur, synchronous machines within these groups move coherently. This is the basis for the equivalence of the two groups of synchronous machines under 
consideration and the representation of the original EPS as a two-machine equivalent. The article formulates an approach to assessing the stability of EPS in relation to the EPS under study using the area method.

\section{References}

1. V.A. Venikov. Transient electromechanical processes in electrical systems. M .: Higher school, 1985.

2. P. Kundur. Power system stability and control. New York: McGraw Hill, 1994.

3. L.D. Landau, E.M. Lifshits. Mechanics / Vol. 1: Theoretical physics. Moscow: Nauka, 1965.

4. N.N. Lizalek, V.F. Tonyshev. Forecasting and identification of instability in electric power systems. Novosibirsk: Novosib. state acad. water transp., 2013.

5. N.A. Abramenkova, N.I. Voropai, T.B. Zaslavskaya. Structural analysis of electric power systems (In the problems of modeling and synthesis). Novosibirsk: Nauka, 1990. 